\title{
A review of biolubricants in drilling fluids: Recent research, performance, and applications
}

\begin{abstract}
Lubricant additives are added to drilling fluids to lower the drag and torque between the drill strings and rock formation. They impart lubricating properties into two moving surfaces contacts under extreme temperature and pressure conditions. Currently, there is a significant interest in developing biolubricants derived from organic sources. Biolubricants offer several valuable friction-reducing physicochemical properties, including high lubricity, wide viscosity range, low pour point, high flash point, high thermal stability, and biodegradability when applied in drilling operation. This study reviews the application of general lubricants in drilling fluids and the potential of biolubricants derived from vegetable oils in exceeding the performance of hydrocarbon and mineral-based lubricants. Overall, biolubricants possess most of the physicochemical properties required as a lubricant for drilling fluids. The utilization of organic ester-based biolubricant might result in faster and deeper drilling, lower bioaccumulation and high biodegradability characteristic, less waste volume, and reduced in overall operation cost. Among the available biolubricants, polyol esters of vegetable oils demonstrate the most suitable lubricants for many drilling conditions, even though their application is still very limited.
\end{abstract}

Keywords: Biolubricant; Drilling fluid lubricant; Polyol ester; Lubricity enhancer; Differential sticking 